\title{
Three-dimensional agent-based modeling of the human epidermal structure and barrier function with realistic cell morphology
}

Sis 0

(1) 00

ESE

ohnsonafolunson

Thomas Sütterlin ${ }^{1}$, Erika Tsingos ${ }^{1}$, Jalil Bensaci ${ }^{2}$, Georgios N. Stamatas ${ }^{2},{ }^{*}$ and Niels Grabe ${ }^{1}$

${ }^{1}$ Hamamatsu TIGA Center, BioQuant, Heidelberg University, Heidelberg, Germany

${ }^{2}$ Emerging Science \& Innovation, Johnson \& Johnson Santé Beauté France, Issy-les-Moulineaux, France

In this work, we created a three-dimensional computational model of the human epidermal structure and water barrier function, able to realistically represent cell morphology and tissue organization. We developed a biomechanical model (BM) using ellipsoid cell shapes corresponding to the differentiation state of the keratinocytes. We used the modeling and simulation EPISIM platform to create a cell behavioral model (CBM) integrated with the BM that considers cell proliferation, differentiation, and desquamation processes, as well as regulatory interactions between water and calcium flow within the tissue.

EPISIM Platform

- Multiscale, multicellular.

- Graphical modelling system (Modeller).

- Agent-based simulation environment (Simulator).

Each EPISIM-based model is composed of at least a cell behavioural and a biomechanical model (CBM and BM). The BM covers all spatial and biophysical cell properties.

EPISIM Modeller allows to graphically construct CBMs, i.e. models of cellular decisions, through process diagrams. These can be automatically translated into executable code, which is loaded in EPISIM Simulator to simulate an agent-

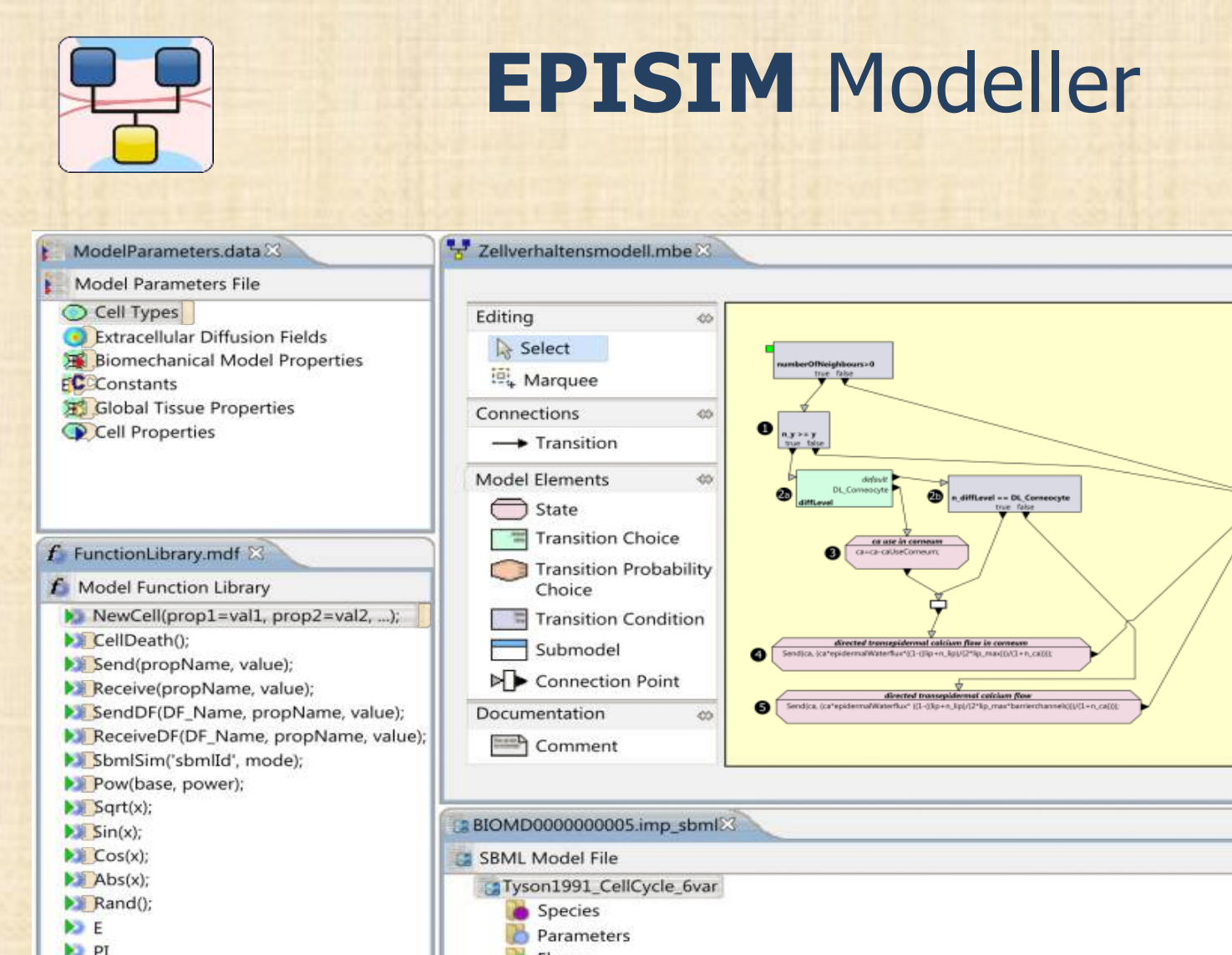
based tissue.

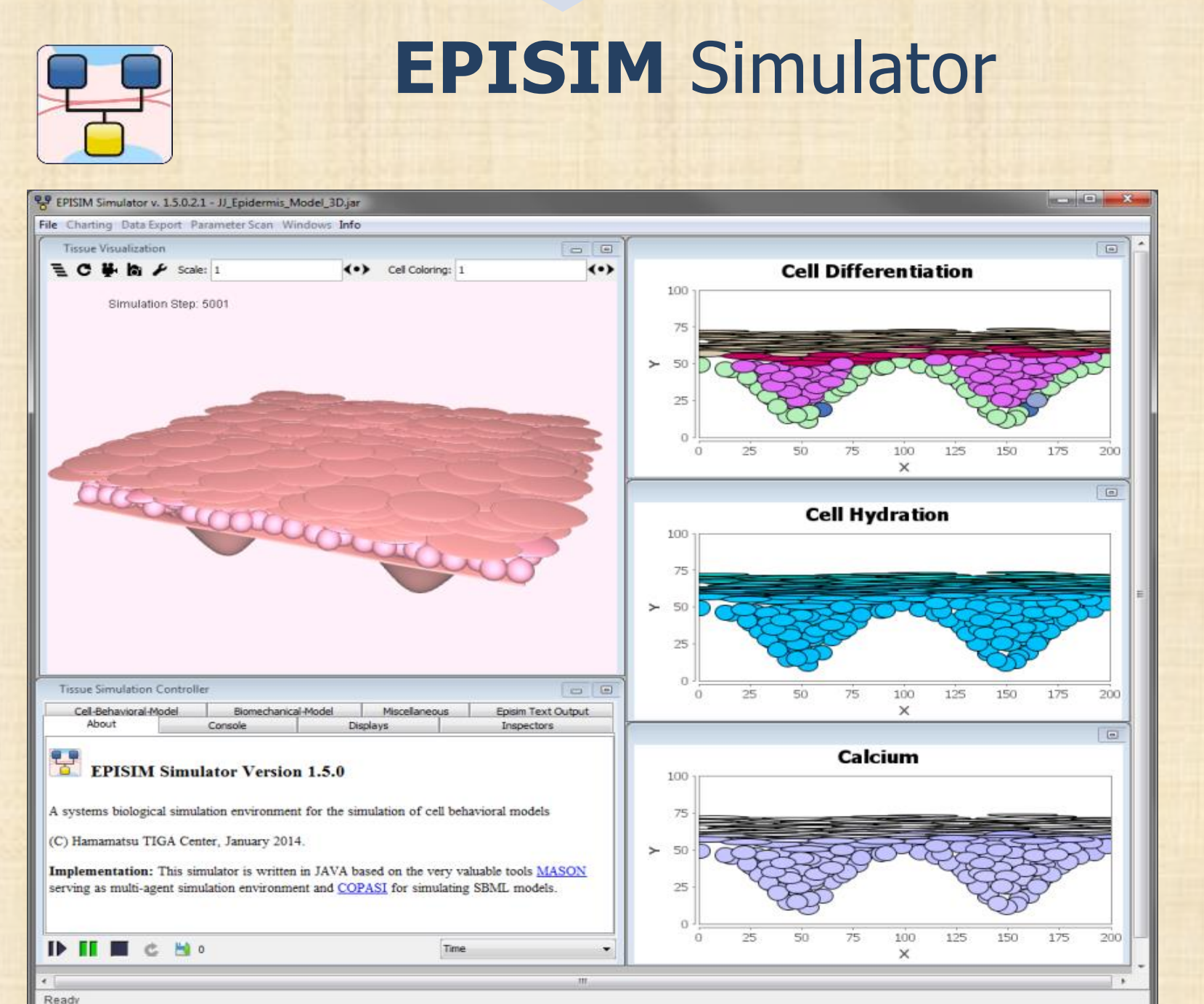

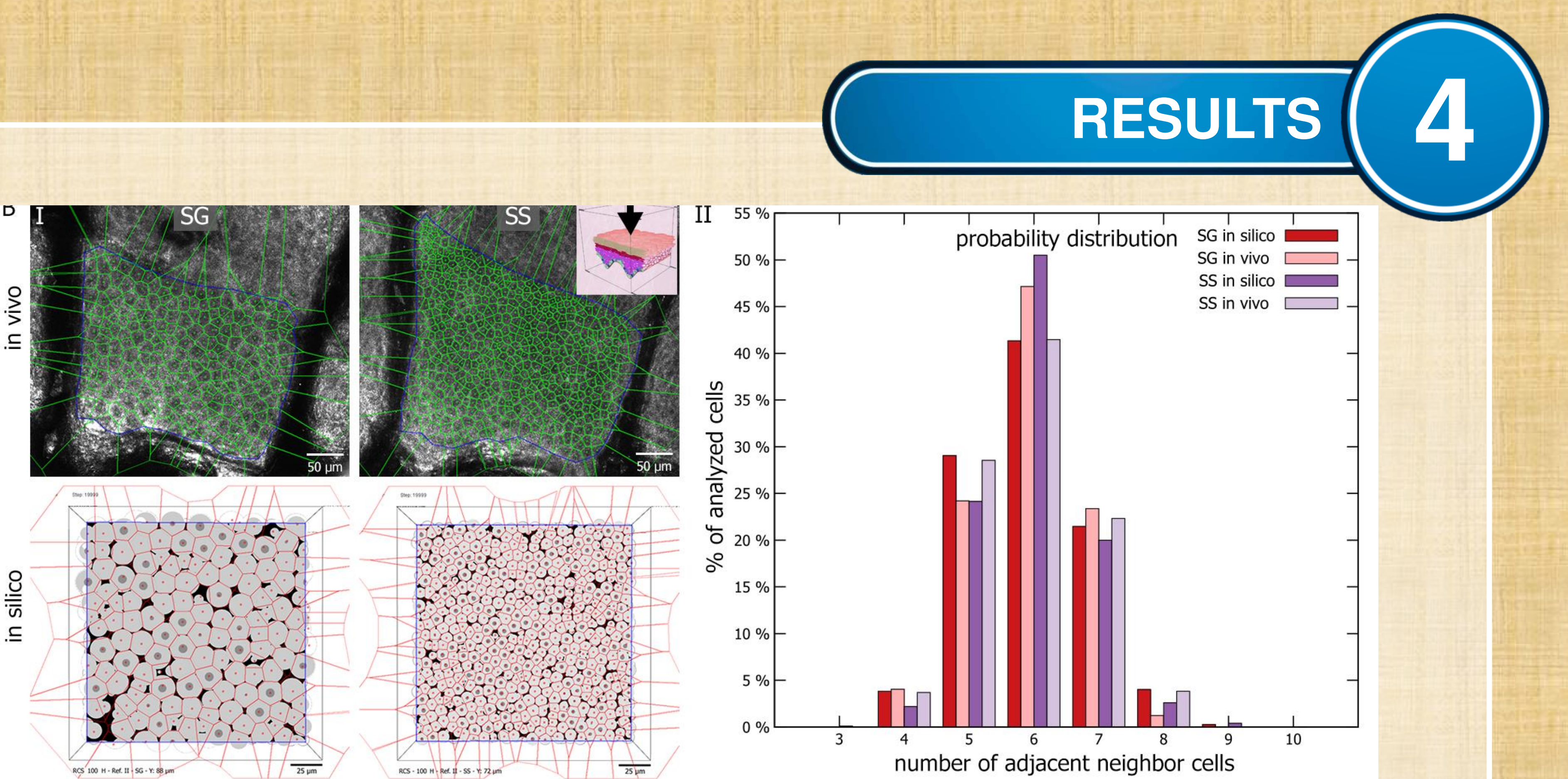

Figure 2: The epidermal topology is recreated as an emerging property of the model.
Starting from a small number of stem cells, the model can reach a steady state of a self-organized, continuously selfrenewing, stratified epidermis (Fig 1). Topological analysis showed that the model-predicted probability distribution of nearest neighbours per cell follows closely the distribution observed in vivo (Fig 2). Water and calcium gradients, closely resembling those of the physiological state, are generated as emerging properties of the model (Fig 3 ).
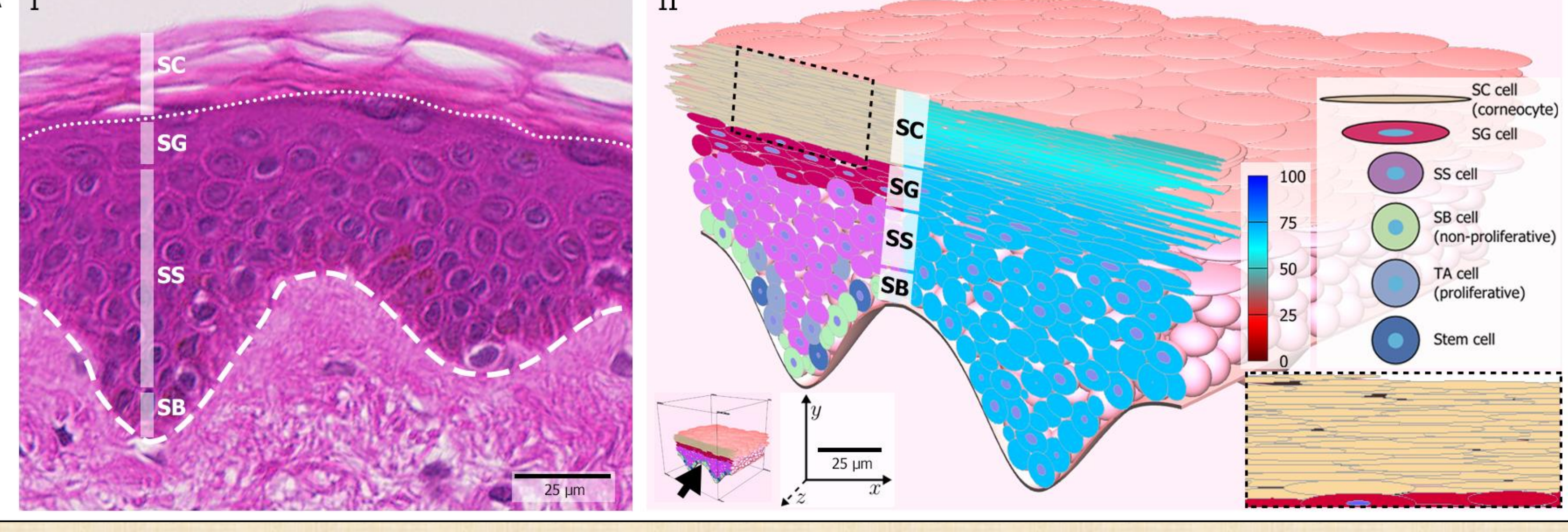

Figure 1: The epidermal layered structure $(\mathrm{I})$ is recreated as an emerging property of the model (II). The water concentration gradients can be visualized (II).
RESULTS

We then tested the model's behavior by varying the ambient humidity parameter and found that once in homeostasis, the epidermal barrier can efficiently buffer changes in humidity levels. Tape stripping experiments can be simulated in silico by removal of SC, which led initially to a homeostatic imbalance of the calcium and water gradients. This provoked an emergency response, accelerating differentiation process resulting in a temporary overshoot of the epidermal thickness, before its return to a homeostatic state (Fig 4).
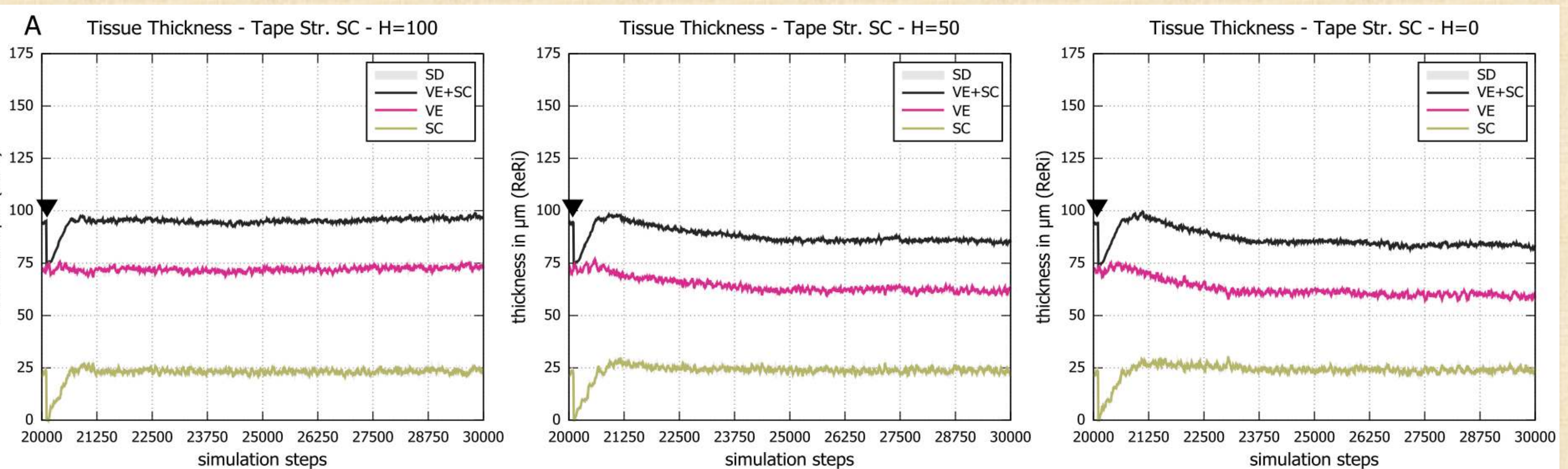

Figure 4: Tissue thickness overshoot after tape stripping, in three different moisturization level environments

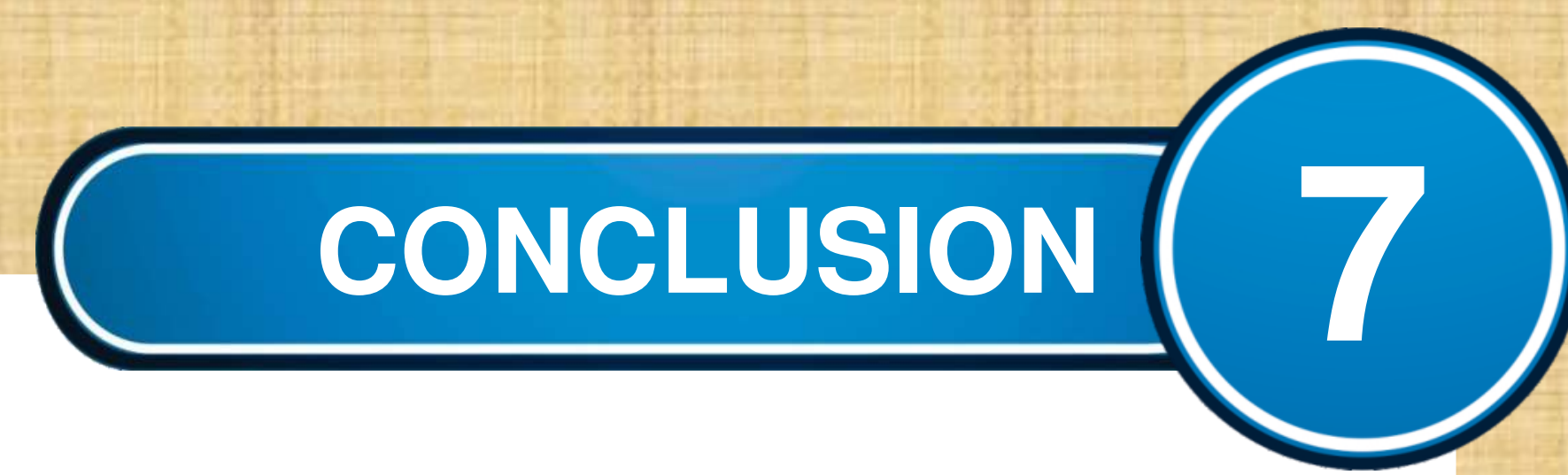

The presented model reproduces essential characteristics of the epidermal homeostasis, barrier, and hydration.

This work paves the way to computationally investigate how an impaired epidermal function precipitates disease. 